\title{
Sudden death in young people due to hypertrophic cardiomyopathy
}

\author{
A. L. GOURDIE, C. E. ROBERTSON \& A. BUSUTTIL* \\ Department of Accident and Emergency Department, Royal Infirmary of Edinburgh, and $\vec{\circ}$ \\ *Department of Pathology, University Medical School, Edinburgh, Scotland

\section{INTRODUCTION}

Hypertrophic cardiomyopathy is a recognized cause of sudden death in young people. $A_{-}^{\stackrel{\circ}{3}}$ series of cases presenting to a teaching hospital accident and emergency department are $\infty$ presented. Emphasis is placed upon the clinical presentation, diagnosis and manage- $\frac{\mathbb{O}}{0}$ ment of the condition.

\section{CASE HISTORIES}

Case I

A 19-year-old Caucasian woman with no previous medical history of note collapsed suddenly in the street. On arrival at the scene of the ambulance crew, she was found to be in cardiac arrest and was given basic life support during transportation to hospital. Following arrival in the accident and emergency department, she was found to be in ventricular fibrillation. Following defibrillation, a stable sinus rhythm ensued with 3 good cardiac output. A 12-lead ECG showed characteristic features of left ventricular hypertrophy and 'splintering' of the QRS complex (Fig. 1). The pulse was noted to have a jerky quality and a systolic murmur was audible maximally at the lower left sternal edge. Echocardiography confirmed the clinical diagnosis of hypertrophic obstructive cardiomyopathy. Despite maintaining adequate perfusion and a stable rhythm, the patient did not regain consciousness and died 3 days later. At post-mortem, classical features of hypertrophic obstructive cardiomyopathy were found (Fig. 2).

Correspondence: C.E. Robertson, Consultant, Accident and Emergency Department, Royal Infirmary of Edinburgh, Lauriston Place, Edinburgh EH3 9YW, Scotland. 


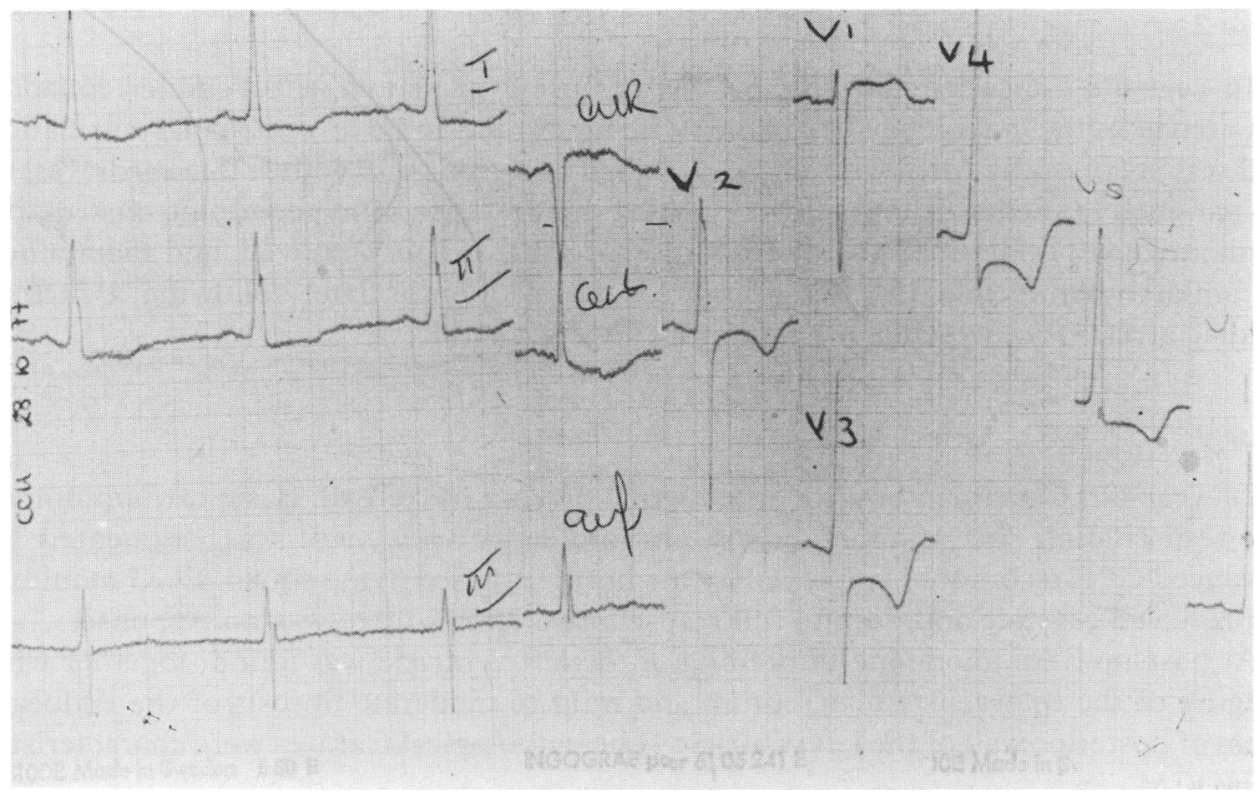

Fig. 1 A 12-lead ECG showing characteristic features of left ventricular hypertrophy and 'splintering' of the QRS complex.

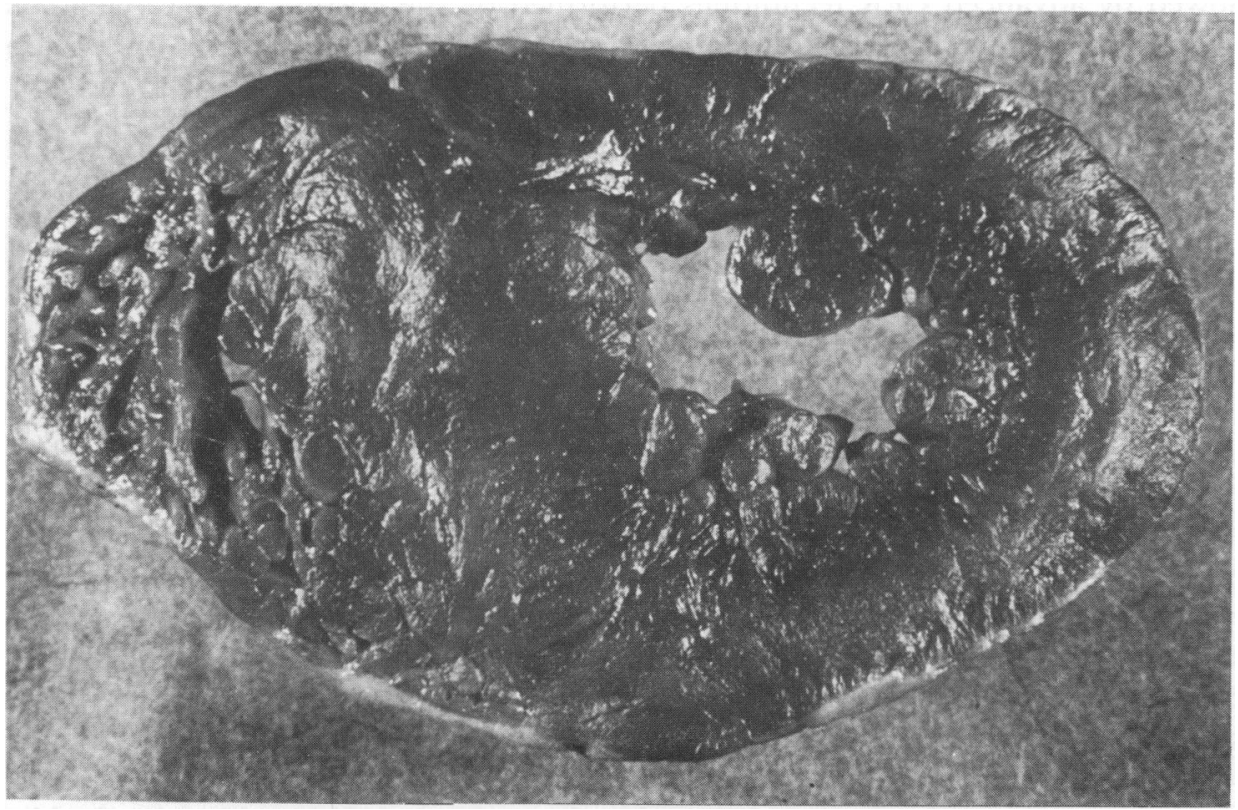

Fig. 2 Classical features of hypertrophic obstructive cardiomyopathy. 
Case 2

A 16-year-old Caucasian male (the brother of Case I) presented with a sudden collapse. On arrival in the emergency department, he was found to be in ventricular fibrillation and was successfully cardioverted. He regained consciousness with stable cardiovascular parameters. Echocardiography confirmed the presence of hypertrophic obstructive cardiomyopathy. He was subsequently discharged from hospital well, and maintained on antiarrhythmic therapy, but presented several months later following a further cardiac arrest from which resuscitation was unsuccessful.

\section{Case 3}

A 14-year-old Caucasian boy collapsed while playing basketball. Basic cardiopulmonary resuscitation was commenced by an ambulance crew, and was augmented by advanced CPR techniques employed by the hospital-based flying squad. ECG monitoring revealed asystole and despite full resuscitative efforts there was no response.

At post-mortem, moderate thickening of the left ventricle was found, together with bulging of the interventricular septum and mild to moderate fibrosis of the endocardium of posterior wall of the left ventricle. The pathological features were characteristic of HOCM.

\section{Case 4}

A 14-year-old Caucasian boy who was a keen boxer was found unrousable in bed a home. Basic life support commenced by the initial ambulance crew on the scene wa⿳亠 followed by advanced CPR techniques performed by the hospital-based flying squađ but without response. At post-mortem the heart weighed $500 \mathrm{~g}$, and gross hypertrophy of the interventricular septum and left ventricle, typical of HOCM, was found.

\section{Case 5}

A 35-year-old Caucasian female collapsed suddenly while on a training run. Basic life support was instituted by trained bystanders within 2-3 min and continued in transit to hospital. On arrival in the resuscitation room, the ECG trace revealed fine ventricular fibrillation. This was resistant to DC cardioversion. Closed chest compression together with positive-pressure ventilation via an endotracheal tube was continued together with standard drug therapy according to Resuscitation Council (UK) guidelines. No clinical response was elicited to these measures. Accordingly internal cardiac massage was instituted following thoracotomy in the resuscitation room. During direct cardiac compression, the myocardium was noted to be stiff and unyielding. After 5 min of open cardiac massage, intermittent pulsation was noted, but despite optimization of acid-base status and inotropic support this was not sustained. At post-mortem, the heart weighed $435 \mathrm{~g}$. There was thickening of the interventricular septum $(3 \mathrm{~cm})$ and the left ventricular wall was concentrically hypertrophied. There were areas of patchy fibrosis throughout the myocardium.

On further enquiry, it was established that the patient's father and brother had both died before 40 years of age with sudden 'cardiac' death. 


\section{DISCUSSION}

Hypertrophic obstructive cardiomyopathy (HOCM) was first described in 1951 by Brock and subsequently characterized by Goodwin (1960) and Braunvald et al., (1964). It is a genetically determined disease of cardiac muscle with autosomal dominant transmission (Emanuel \& Withers, 1983). The myocardium, especially the interventricular septum is hypertrophied and the muscle fibres are shortened, thickened and arranged in circular formations together with associated fibrous tissue. On electron microscopy, the myofibrils are found to be disorganized and no longer exhibit their normal parallel arrangement. While the coronary vessels are generally normal, the mitral valve papillary muscles are often greatly hypertrophied (Maron et al., 1974, 1978).

As a consequence of these pathological changes, a number of functional abnormalities ensue. The principal dysfunction occurs during diastole when, because of the abnormal muscle function within the ventricles, relaxation is irregular and as a consequence, diastolic filling is delayed. Systole is normally rapid and strong (Maron et al., 1987).

Commonly exhibited symptoms include angina on exertion, syncope or nearsyncope, dyspnoea and tiredness. However symptomatology does not correlate well with either the severity or progression of the disease process (Maron et al., 1987). The risk of sudden cardiac death is well recognized and is the most important problem in patient management. Patients with proven episodic ventricular arrhythmias during electrocardiographic monitoring have an even higher risk of sudden death (Maron et al., 1981). The mortality for premature death from HOCM is approximately $2-3 \%$ per annum, the great majority of such deaths are sudden and unexpected.

Sudden death may be due to either a primary ventricular arrhythmia or from haemodynamic changes relating to an acute increase in outflow obstruction (Maron $e t$ $a l ., 1987)$. Several arrhythmias have been described in HOCM including supraventricular and ventricular tachyarrhythmias, and complete heart block. The occurrence of ventricular tachycardia in an individual patient seems to be the most predictive abnormality associated with sudden death. Indications for therapy in asymptomatic patients are controversial, while for symptomatic individuals the choice of antiarrhythmic agents or for surgical therapy is wide. For those patients proven to have ventricular arrhythmias by electrocardiographic monitoring or during electrophysiological studies, amiodarone is probably the drug of choice, although beta adrenergic blockers and calcium antagonists have also been advocated (McKenna et al., 1985). The commonest surgical intervention has been ventricular septal myotomy-myectomy to relieve the dynamic obstruction to left ventricular outflow. Mitral valve replacement has also been advocated in selected cases and will relieve any subaortic gradient.

Symptomatic improvement occurs in almost three-quarters of patients following surgery with improved exercise tolerance. However there is no evidence of prolonged lifespan, and the operative mortality is up to $8 \%$ (Maron et al., 1987). Cardiac transplantation may also have a role in management particularly when cardiac failure predominates.

Many patients with HOCM die suddenly, during of after sudden exertion. It is of note that Cases 1 and 4 were keen competitive sportspersons while Cases 3 and 5 died while undertaking physical activity. Advice with regard to the avoidance of strenuous or 
competitive physical activities is commonly given and close cardiological follow-up is $\frac{\pi}{0}$ mandatory for such cases (Maron et al., 1980, 1982).

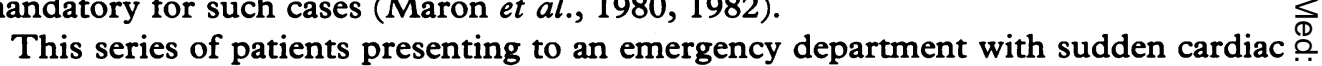
arrest indicates the need for awareness of the condition in young people. Patients $\overrightarrow{\mathrm{F}}$ presenting with unexplained episodes of syncope, collapse or chest pain require $\frac{f}{0}$ appropriate clinical, electrocardiographic and echocardiographic assessment. The dia- 흠 gnosis should be considered particularly in young patients presenting in cardiac arrest $\frac{\bar{\omega}}{\vec{D}}$ in whom initial resuscitation is successful. For those patients subsequently found to be $\stackrel{\mathbb{Q}}{\varrho}$ suffering from HOCM, either at clinical follow-up or post-mortem, screening of other family members is indicated and appropriate advice and therapy offered.

\section{REFERENCES}

Brock R. (1957) Functional obstruction of the left ventricle. Guys Hospital Gazette 106, 221-38.

Goodwin J. F., Hollman A., Cleland W. P. \& Teare D. (1960) Obstructive cardiomyopathy simulating aortic stenosis. British Heart fournal 22, 403-14.

Braunvald E., Lambuew C. T., Rockoff S. D., Ross J. Jr. \& Morrow A. C. (1964) Idiopathic hypertrophic $\vec{c}$ subaortic stenosis. 1. A description of the disease based upon an analysis of 64 patients. Circulation $\frac{\mathbb{D}}{8}$ 30(Suppl IV), 3-119.

Emanuel R. \& Withers R. (1983) The Cardiomyopathies in Principle of Practice of Medical Genetics Volume 2. Churchill Livingstone, Edinburgh.

McKenna W. J., Oakley C. M., Krikler D. M. \& Goodwin J. F. (1985) Improved survival with amiodarone i̊ $\overrightarrow{0}$ patients with hypertrophic cardiomyopathy and ventricular tachycardia. British Heart fournal 53, 412-

Maron B. J., Borrow R. O., Cannon R. O. et al., (1987) Hypertrophic cardiomyopathy-interrelations gf. clinical manifestations, pathophysiology and therapy. New England fournal of Medicine 316, 844-52.

Maron B. J., Ferrans V. J., Henry W. L. et al., (1974) Differences in distribution of myocardial abnormalities in patients with obstructive and non-obstructive asymmetric septal hypertrophy (ASH): light and electron microscopic findings. Circulation 50, 436-46.

Maron B. J., Roberts W. C., Edwards J.E. et al., (1978) Sudden death in patients with hypertrophic $\stackrel{\mathbb{Q}}{\varrho}$ cardiomyopathy: characterization of 26 patients without functional limitation. American fournal of $\overrightarrow{\vec{B}}$ Circulation 41, 803-10.

Maron B. J., Roberts W. C. \& Epstein S. E. (1982) Sudden death in hypertrophic cardiomyopathy: a profile of 78 patients. Circulation 65, 1388-94.

Maron B. J., Roberts W. C., McAllister H. A. et al., (1980) Sudden death in young athletes. Circulation 62, 218-29.

Maron B. J., Savage D. D., Wolfson J. K. \& Epstein S. E. (1981) Prognostic significance of $24 \mathrm{~h}$ ambulatory electrocardiographic monitoring in patients with hypertrophic cardiomyopathy-a prospective study. American fournal of Cardiology 48, 252-7. 